\title{
Como os hospitais universitários estão enfrentando a pandemia de COVID-19 no Brasil?
}

How are university hospitals coping with the COVID-19 pandemic in Brazil? ¿Cómo están enfrentando la pandemia de COVID-19 los hospitales universitarios en Brasil?

José Luís Guedes dos Santos ${ }^{1}$ iD https://orcid.org/0000-0003-3186-8286

Gabriela Marcellino de Melo Lanzoni ${ }^{1}$ id https://orcid.org/0000-0001-5935-8849

Maria Fernanda Baeta Neves Alonso da Costa ${ }^{1}$ is https://orcid.org/0000-0002-2763-8050

Juanah Oliveira Debetio ${ }^{1}$ io https://orcid.org/0000-0001-5894-2154

Leonardo Pereira de Sousa ${ }^{1}$ io https://orcid.org/0000-0003-2047-5639

Lucas Soares dos Santos ${ }^{1}$ iD https://orcid.org/0000-0002-2953-8651

Tatiane Boeing Marcelino ${ }^{1}$ id https://orcid.org/0000-0002-6289-5263

Ana Lúcia Schaefer Ferreira de Mello ${ }^{1}$ io https://orcid.org/0000-0001-9591-7361

\section{Como citar:}

Santos JL, Lanzoni GM, Costa MF, Debetio JO, Sousa LP, Santos LS, et al. Como os hospitais universitários estão enfrentando a pandemia de COVID-19 no Brasil?. Acta Paul Enferm. 2020;33:APE20200175.

DOI

http://dx.doi.org/10.37689/actaape/2020A001755

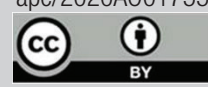

Descritores

Coronavirus; Infeccões por coronavírus; Pandemias; COVID-19; Gestão em saúde; Hospitais universitários

Keywords

Coronavirus: Coronavirus infections; Pandemics; COVID-19; Health management; Hospitals, university

Descriptores Coronavirus; Infecciones por coronavirus: Pandemias: COVID-19; Gestión en salud; Hospitales universitarios

\section{Submetido 8 de Julho de 2020 \\ Aceito \\ 19 de Agosto de 2020}

Autor correspondente José Luís Guedes dos Santos E-mail: jose.santos@ufsc.br

\section{Resumo}

Objetivo: Identificar as ações que os hospitais universitários federais estão desenvolvendo no enfrentamento da pandemia de COVID-19 no Brasil.

Métodos: Estudo documental, a partir de informações disponibilizadas nos sites oficiais de 44 hospitais universitários da rede federal de ensino do Brasil. 0 levantamento foi realizado nos meses de abril e maio de 2020. Os excertos foram agrupados por similaridade, considerando as dimensões Assistência, Gestão, Extensão, Ensino e Pesquisa. Para análise dos dados, utilizou-se estatística descritiva.

Resultados: Identificaram-se 495 ações para 0 enfrentamento da pandemia de COVID-19, distribuídas entre Assistência (38,99\%), Gestão (37,58\%), Extensão (16,16\%) e Ensino e Pesquisa (7,27\%). Na Assistência, destacou-se a suspensão de consultas não relacionadas à COVID-19 e cirurgias eletivas, bem como mudanças em rotinas com reforço às questões de biossegurança para evitar transmissão do vírus. № âmbito da Gestão, ocorreu contratação de novos profissionais e capacitação das equipes de saúde. Na dimensão Extensão, foram desenvolvidos principalmente materiais educativos sobre medidas de prevenção da COVID-19. Em relação ao Ensino e Pesquisa, houve suspensão de atividades educativo-formativas e sobressaiu-se 0 engajamento dos hospitais no desenvolvimento de pesquisas sobre 0 tema.

Conclusão: 0s hospitais universitários estão reorganizando 0 atendimento e buscando meios para prover as condições necessárias para o cumprimento da sua missão no contexto do Sistema Único de Saúde, a partir de ações de assistência, gestão, extensão, ensino e pesquisa.

\section{Abstract}

Objective: To identify the actions that federal university hospitals are developing to tackle the COVID-19 pandemic in Brazil.

Methods: Documentary study based on information available on official websites of 44 university hospitals in the federal teaching network in Brazil. The survey was conducted in April and May 2020. The excerpts were grouped by similarity, considering the Care, Management, Extension, Teaching and Research dimensions. Descriptive statistics was used for data analysis.

Results: The 495 actions identified to cope with the COVID-19 pandemic were distributed among Care (38.99\%), Management (37.58\%), Extension (16.16\%) and Teaching and Research (7.27\%). In Care, the highlights were the suspension of consultations not related to COVID-19 and elective surgeries, and changes in routines with focus on biosafety issues to prevent virus transmission. In Management, new professionals were hired and health teams were trained. In the Extension dimension, educational materials were developed, mainly on COVID-19 prevention measures. In relation to Teaching and Research, educational-training activities 
were suspended and the engagement of hospitals in the development of research on the subject stood out.

Conclusion: University hospitals are reorganizing care and looking for ways to provide the necessary conditions to fulfill their mission within the National Health System context, based on care, management, extension, teaching and research actions.

\section{Resumen}

Objetivo: Identificar las acciones que los hospitales universitarios federales están llevando a cabo para enfrentar la pandemia de COVID-19 en Brasil.

Métodos: Estudio documental, a partir de información disponible en los sitios web oficiales de 44 hospitales universitarios de la red educativa federal de Brasil. La recolección fue realizada en los meses de abril y mayo de 2020. Los fragmentos se agruparon por similitud, considerando las dimensiones Atención, Gestión, Extensión, Enseñanza e Investigación. Se utilizó estadística descriptiva para analizar los datos.

Resultados: Se identificaron 495 acciones para el enfrentamiento de la pandemia de COVID-19, distribuidas entre Atención (38,99 \%), Gestión (37,58 \%), Extensión (16,16 \%) y Enseñanza e Investigación (7,27 \%). En Atención, se destacó la suspensión de consultas no relacionadas con la CovID-19 y cirugías electivas, así como cambios de rutinas con un refuerzo en las cuestiones de bioseguridad para evitar la transmisión del virus. En el ámbito de la Gestión, se contrataron nuevos profesionales y se capacitó a los equipos de salud. En la dimensión Extensión, se creó principalmente material educativo sobre medidas de prevención de la COVID-19. Con relación a la Enseñanza e Investigación, se suspendieron las actividades educativas y se destacó el compromiso de los hospitales en el desarrollo de investigaciones sobre el tema.

Conclusión: Los hospitales universitarios están reorganizando la atención y buscando los medios para ofrecer las condiciones necesarias para cumplir su misión en el contexto del Sistema Único de Salud, a partir de acciones de atención, gestión, extensión, enseñanza e investigación.

\section{Introdução}

No final do ano de 2019, surgiu uma nova doença provocada pelo coronavírus na cidade de Wuhan, região central da China, causada pelo vírus SARSCoV-2, denominada COVID-19 (Doença por Coronavírus 2019). Em cerca de dois meses, a COVID-19 disseminou-se rapidamente pela Ásia e atingiu todos os continentes, tornando-se o mais importante problema mundial de saúde pública dos últimos 100 anos. Diante da magnitude da infecção, a Organização Mundial da Saúde (OMS) atribuiu à doença a classificação de pandemia em março de $2020 .^{(1-3)} \mathrm{Na}$ América Latina, o primeiro caso de COVID-19 foi confirmado em 25 de fevereiro de 2020, no Brasil. ${ }^{(4)}$

A COVID-19 é potencialmente fatal, por se tratar de uma doença nova com comportamento imprevisível e rápida disseminação. O SARS-CoV-2 é altamente transmissível por gotículas e contato, principalmente em locais fechados. Um indivíduo com infecção pelo novo coronavírus transmite para outras duas ou três pessoas, dependendo das condiçóes ambientais. Além disso, ainda não foram identificados tratamentos farmacológicos comprovadamente eficazes e/ou vacina específica. ${ }^{(3-5)}$

O tratamento da doença é sintomático, sendo que a atenção hospitalar tem um papel fundamental na realização de cuidados intensivos aos pacientes que apresentam sintomas graves. Dessa forma, tem-se observado mundialmente que a COVID-19 eleva a demanda por atendimento nas unidades hospitalares, tornando necessária a reorganização dos processos de cuidado e o desenvolvimento de estratégias gerenciais para o enfrentamento da pandemia. ${ }^{(6,7)}$

No Brasil, os Hospitais Universitários Federais (HUFs) destacam-se no atendimento a pacientes com COVID-19 como centros de referência de média e alta complexidade para o Sistema Único de Saúde (SUS). Além disso, têm significativo papel na formação de recursos humanos em saúde e no apoio ao ensino, à pesquisa e à extensão nas instituiçôes de ensino superior as quais estão vinculados. ${ }^{(8,9)}$

Nenhum país estava preparado para enfrentar uma epidemia de COVID-19, que tem gerado impactos negativos nas áreas social, econômica, na saúde física e mental das populaçôes, bem com, na capacidade assistencial dos sistemas de saúde. No caso dos hospitais, as açôes de enfrentamento à COVID-19 podem variar conforme a gravidade dos pacientes, o perfil assistencial do hospital e a epidemiologia local da doença. De modo geral, observa-se que os principais desafios dos hospitais envolvem a ampliação de leitos de terapia intensiva, capacitação dos profissionais e aquisição de equipamentos de proteção individual em qualidade e quantidades adequadas. ${ }^{(3,6,10)}$ Essas açóes devem ser dinâmicas e adaptativas ao atual conhecimento do vírus e a evolução epidêmica da doença. Desse modo, o sucesso no processo de gestáo hospitalar desse quadro de emergência de saúde pública de- 
manda oferta de cobertura assistencial com foco na avaliação, prevenção e tratamento dos casos diagnosticados. ${ }^{(5,11)}$

Portanto, o enfrentamento da pandemia de COVID-19 remente a um contexto ainda permeado de desafios e que demanda o planejamento de políticas e práticas gerenciais eficazes para a provisão de condiçóes estruturais para o cuidado em saúde nos cenários hospitalares. Para isso, são necessários estudos visando à identificaçáo do que tem sido feito em resposta à pandemia e, principalmente, apoiar as decisóes a serem tomadas para o enfrentamento desse momento crítico.

A partir do panorama exposto, delineou-se como questão de pesquisa: Como os hospitais universitários federais estão enfrentando a pandemia de COVID-19 no Brasil? Este estudo teve por objetivo identificar as açôes que os hospitais universitários federais estão desenvolvendo no enfrentamento da pandemia de COVID-19 no Brasil.

\section{Métodos}

Trata-se de uma pesquisa documental, quantitativa, realizada a partir da consulta aos sites eletrônicos dos HUFs brasileiros. No Brasil, a rede de hospitais universitários federais é formada por 50 hospitais vinculados a 35 Instituiçôes Federais de Ensino (IFEs). ${ }^{(8)}$

A coleta de dados foi realizada por quatro pesquisadores individualmente, após ajuste dos parâmetros de busca e extração das informações dos sites. Os sites eletrônicos de todos os HUFs foram acessados entre 27 de abril a 01 de maio de 2020 . Duas IFEs possuíam dois HUFs cada, mas reunidos em um mesmo complexo hospitalar. Dessa forma, foram 48 os sites consultados. Além disso, foram excluídos dois HUFs que não tinham publicaçôes relativas à COVID-19 em seus sites e dois HUFs cujos sites estavam em manutenção. Todos os HUFs tinham site independente das IFEs as quais estavam vinculados. Assim, a busca ficou limitada aos seus próprios endereços eletrônicos de origem. A amostra final do estudo foi composta por 44 hospitais, conforme ilustra a figura $1 .^{(8)}$

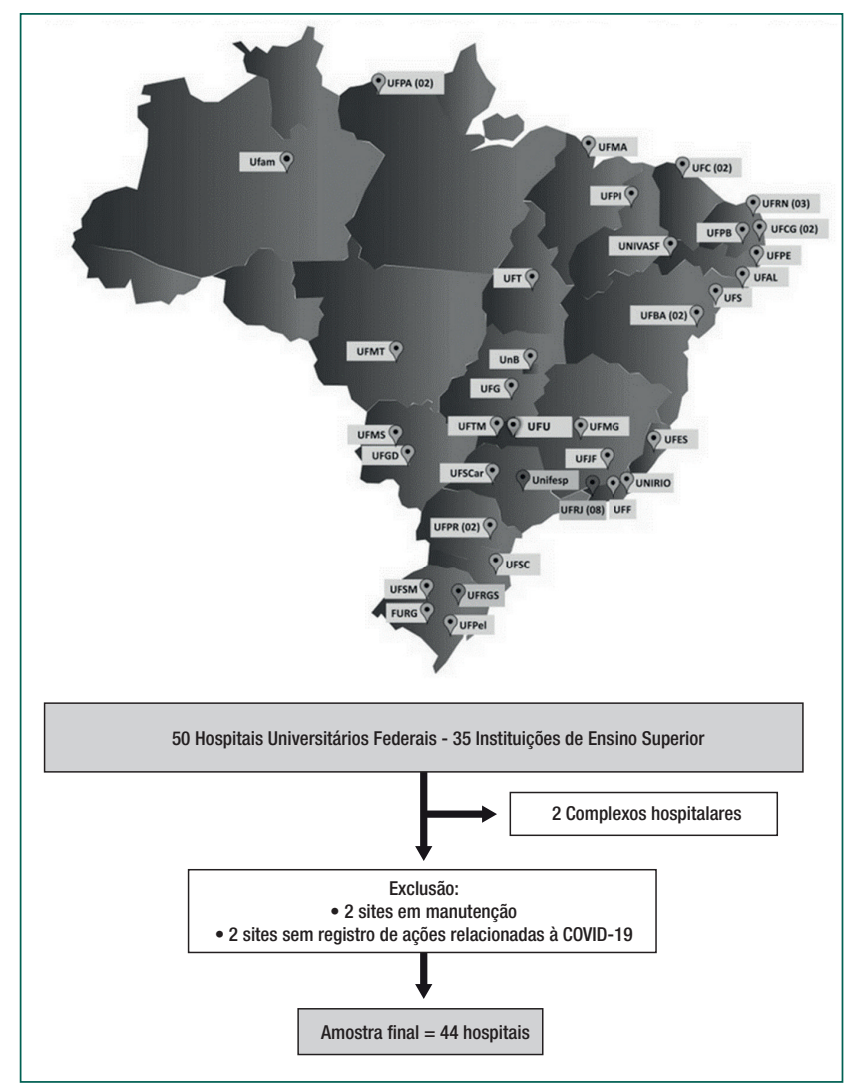

Fonte: Figura adaptada da Empresa Brasileira de Serviços Hospitalares. Brasil, 2020.(8)

Figura 1. Fluxo de seleção dos hospitais universitários

Para caracterização dos hospitais, foi consultado o Cadastro Nacional de Estabelecimentos de Saúde (CNES), disponibilizado pelo DATASUS, em regime de domínio público. Foram extraídas informações sobre o quantitativo de profissionais, a exclusividade do atendimento pelo SUS, o número de leitos gerais e o quantitativo de leitos de terapia intensiva para pacientes adultos.

A coleta de dados sobre as ações realizadas para o enfrentamento da pandemia COVID-19 focou na divulgação de notícias e informaçôes gerais nos sites sob forma de textos. Os excertos identificados foram copiados para uma planilha de dados no software Word $^{\circledR}$, segundo cada hospital.

Para a análise dos dados, definiram-se, a priori, quatro dimensões: Gestão, Assistência, Extensão, Pesquisa e Ensino, as quais são os principais eixos de atuação dos HUFs. ${ }^{\left({ }^{8,9)}\right.} \mathrm{O}$ conteúdo retirado dos sites foi classificado em cada dimensão. Houve duas reunióes entre os autores para discussão e estabelecimento de consenso sobre melhor adequação e 
Tabela 1. Caracterização dos hospitais $(n=44)$ estratificados por região

\begin{tabular}{|c|c|c|c|c|c|c|c|c|c|c|c|c|c|c|c|c|}
\hline \multirow[t]{2}{*}{ Regiões } & \multicolumn{2}{|c|}{$\begin{array}{l}\text { Atendimento } \\
\text { exclusivo pelo } \\
\text { SUS }^{*}\end{array}$} & \multicolumn{2}{|c|}{$\begin{array}{l}\text { Classificação do } \\
\text { Hospital }^{*}\end{array}$} & \multicolumn{4}{|c|}{$\begin{array}{l}\text { Número de } \\
\text { Profissionais* }\end{array}$} & \multicolumn{4}{|c|}{ Número de leitos gerais* } & \multicolumn{4}{|c|}{ Número de leitos de UTI** } \\
\hline & Sim & Não & Especializado & Geral & $\begin{array}{l}\text { Até } \\
700\end{array}$ & $\begin{array}{l}701- \\
1400\end{array}$ & $\begin{array}{c}1401- \\
2100\end{array}$ & $>2101$ & $\begin{array}{l}\text { Até } \\
100\end{array}$ & $\begin{array}{c}101- \\
200\end{array}$ & $\begin{array}{c}201 \text { - } \\
300\end{array}$ & $>301$ & $\begin{array}{c}\text { Até } \\
10\end{array}$ & $11-20$ & $21-30$ & $>31$ \\
\hline Centro-Oeste e DF & $1(20)$ & $4(80)$ & - & $5(100)$ & - & $2(40)$ & $2(40)$ & $1(20)$ & - & $2(40)$ & $2(40)$ & 1(20) & - & $3(75)$ & - & 1(25) \\
\hline Nordeste & - & $17(100)$ & $4(23,5)$ & $13(76,5)$ & $3(17,6)$ & $5(29,4)$ & $6(35,3)$ & $3(17,6)$ & $4(23,5)$ & $4(23,5)$ & $7(41,2)$ & $2(11,8)$ & $8(50)$ & $2(15,2)$ & $4(25)$ & $2(12,5)$ \\
\hline Norte & $1(50)$ & $1(50)$ & $1(50)$ & $1(50)$ & $1(50)$ & $1(50)$ & - & - & $1(50)$ & - & $1(50)$ & - & $1(100)$ & - & - & - \\
\hline Sudeste & $2(13,3)$ & $13(86,7)$ & $5(33,3)$ & $10(67,7)$ & $5(33,3)$ & $1(6,7)$ & $4(26,7 \%)$ & $5(33,3)$ & $5(33,3)$ & $2(13,3)$ & $4(26,7)$ & $4(26,7)$ & $2(20)$ & $2(20)$ & 2(20) & $4(40)$ \\
\hline Sul & 1(20) & $4(80)$ & - & $5(100)$ & - & - & $2(40)$ & $3(60)$ & - & - & $2(40)$ & $3(60)$ & $2(40)$ & - & 1(20) & $2(40)$ \\
\hline
\end{tabular}

${ }^{*} \mathrm{n}=44 ;{ }^{*} \mathrm{n}=36$

padronização da classificação das açôes nas dimensóes. Os dados foram transferidos para o software Excel $^{\oplus}$. Cada uma das açóes foi contabilizada como unidade de análise para contagem de frequência. Procedeu-se a análise estatística descritiva, por meio de frequência simples e relativa.

Todas as informaçóes utilizadas neste estudo são de domínio público. Assim, não foi necessária apreciação do projeto por Comitê de Ética em Pesquisa.

\section{Resultados}

Os 44 HUFs contabilizam ao total 11.034 leitos gerais e 901 leitos de UTI. A região Nordeste concentra o maior número de hospitais $(38,6 \%)$, porém, a região Sudeste possui a maior quantidade de leitos gerais $(33,81 \%)$ e leitos de UTI $(40,18 \%)$. A quantidade de leitos gerais variou de 18 a 981 e de leitos de UTI, estes em 36 hospitais, de 1 a 101. A maioria das instituiçôes está classificada como hospital geral $(77,3 \%)$ e presta atendimento exclusivo ao SUS $(88,6 \%)$. A tabela 1 apresenta o detalhamento da caracterização dos HUFs estratificados por região.

Identificaram-se 495 açóes desenvolvidas pelos HUFs para o enfrentamento da pandemia de COVID-19, distribuídas entre Assistência $(38,99 \%)$, Gestão (37,58\%), Extensão (16,16\%) e Ensino e Pesquisa (7,27\%). Na dimensão Assistência, destacaram-se açóes relacionadas à suspensão e/ou adiamento de consultas ou atendimentos ambulatoriais, restrição e/ou suspensão de visitas e suspensão e/ou adiamento de cirurgias eletivas. Na dimensão Gestão, as principais estratégias foram capacitação de profissionais para o manejo da COVID-19, contratação emergencial de profissio- nais da saúde e oferta de atendimento psicológico para os trabalhadores (Tabela 2).

Tabela 2. Estratégias assistenciais e gerenciais realizadas pelos hospitais $(n=44)$

\begin{tabular}{|c|c|}
\hline Assistência (n=193) & $\mathrm{n}(\%)$ \\
\hline Suspensão e/ou adiamento de consultas ou atendimentos ambulatoriais & $37(19,17)$ \\
\hline Restrição e/ou suspensão de visitas & $35(18,13)$ \\
\hline Suspensão e/ou adiamento de cirurgias eletivas & $29(15,03)$ \\
\hline Restrição da circulação de acompanhantes pelo hospital & $24(12,44)$ \\
\hline $\begin{array}{l}\text { Definição de fluxograma e protocolos de atendimento para paciente } \\
\text { suspeito de infecção pela COVID-19 }\end{array}$ & $22(11,40)$ \\
\hline $\begin{array}{l}\text { Implementação de videochamadas entre pacientes, familiares e equipe de } \\
\text { saúde }\end{array}$ & $20(10,36)$ \\
\hline Suspensão e/ou adiamento de exames & $16(8,29)$ \\
\hline Emissão de receita e avaliação de laudos através de e-mail & $3(1,55)$ \\
\hline $\begin{array}{l}\text { Mensagens motivacionais nas marmitas dos pacientes internados com } \\
\text { suspeita de COVID-19 }\end{array}$ & $2(1,04)$ \\
\hline $\begin{array}{l}\text { Triagem de acompanhantes e visitantes para detecção de problemas } \\
\text { respiratórios }\end{array}$ & $2(1,04)$ \\
\hline Adoção de atendimento referenciado a casos de urgência e emergência & $2(1,04)$ \\
\hline $\begin{array}{l}\text { Desenvolvimento e distribuição de kits terapêuticos para pacientes em } \\
\text { isolamento. }\end{array}$ & $1(0,52)$ \\
\hline Gestão ( $n=186)$ & $\mathrm{n}(\%)$ \\
\hline Capacitação de profissionais para o manejo da COVID-19 & $36(19,35)$ \\
\hline Contratação emergencial de profissionais da saúde & $34(18,28)$ \\
\hline Oferta de atendimento psicológico para os trabalhadores & $22(11,83)$ \\
\hline $\begin{array}{l}\text { Campanha para a arrecadação de EPIs e doações financeiras para } \\
\text { aquisição de ventiladores pulmonares }\end{array}$ & $14(7,53)$ \\
\hline Elaboração de plano de contingência para enfrentamento de COVID-19 & $13(6,99)$ \\
\hline Campanha de agradecimento e incentivo aos profissionais de saúde & $8(4,30)$ \\
\hline Abertura de unidade específica para pacientes com COVID-19 & $8(4,30)$ \\
\hline Compra de insumos e equipamentos de proteção individual & $8(4,30)$ \\
\hline Disponibilização de alojamento especial para profissionais de saúde & $7(3,76)$ \\
\hline Abertura de novos leitos de UTI para pacientes com COVID-19 & $7(3,76)$ \\
\hline Realização de testes de COVID-19 para profissionais de saúde & $7(3,76)$ \\
\hline Campanha de vacinação de profissionais de saúde contra Influenza & $5(2,69)$ \\
\hline Redistribuição e/ou afastamento de profissionais de grupos de risco & $4(2,15)$ \\
\hline $\begin{array}{l}\text { Organização de equipes exclusivas para atendimento a pacientes com } \\
\text { COVID-19 }\end{array}$ & $3(1,61)$ \\
\hline Distribuição de bisnagas de álcool em gel 70\% para os profissionais & $3(1,61)$ \\
\hline Criação de ambulatório de síndrome gripal para profissionais e alunos & $2(1,08)$ \\
\hline Realização de assembleia geral na instituição sobre COVID-19 & $2(1,08)$ \\
\hline Busca de profissionais voluntários & $1(0,54)$ \\
\hline Campanha para a doação de sangue & $1(0,54)$ \\
\hline Sanitização em áreas de grande circulação & $1(0,54)$ \\
\hline
\end{tabular}

Em relação à Extensão, a principal frente de atuação foi a elaboração de materiais educativos so- 
bre medidas de prevenção da COVID-19. Entre os tópicos abordados, destacaram-se orientaçóes para gestantes e crianças sobre nutrição, atividade física e saúde mental para os pacientes e profissionais. No campo do Ensino e da Pesquisa, houve suspensão das atividades de ensino e pesquisa relacionadas à graduação e pós-graduação. Porém, destaca-se o engajamento no desenvolvimento de novos projetos de pesquisa sobre aspectos epidemiológicos, diagnóstico, prevenção e tratamento da COVID-19 (Tabela 3).

Tabela 3. Estratégias de Extensão, Ensino e Pesquisa $(n=44)$

\begin{tabular}{lc}
\hline Extensão (n=80) & $\mathrm{n}(\%)$ \\
\hline Elaboração de materiais educativos sobre prevenção da COVID-19 & $27(33,7)$ \\
$\begin{array}{l}\text { Desenvolvimento de campanhas para recebimento de doações de recursos } \\
\text { da comunidade }\end{array}$ & $24(30,0)$ \\
$\begin{array}{l}\text { Teleorientação para pacientes e teleconsulta aos profissionais de saúde } \\
\begin{array}{l}\text { Divulgação de dicas e atividades online para comunidade em isolamento } \\
\text { social }\end{array}\end{array}$ & $12(15,0)$ \\
$\begin{array}{l}\text { Suspensão de eventos e interrupção de projetos de extensão } \\
\text { Ensino e Pesquisa (n=36) }\end{array}$ & $7(8,7)$ \\
\hline $\begin{array}{l}\text { Suspensão de atividades de ensino (graduação, pós-graduação) em geral } \\
\text { (teóricas, práticas, estágios), de novos projetos de pesquisa (exceto com foco }\end{array}$ & $\mathbf{n}(\%)$ \\
\hline $\begin{array}{l}\text { em COVID-19), de eventos e adaptação das atividades dos programas de } \\
\text { residência }\end{array}$ & \\
$\begin{array}{l}\text { Desenvolvimento de pesquisas científicas sobre aspectos epidemiológicos, } \\
\text { diagnóstico, prevenção e tratamento da COVID-19 }\end{array}$ & $9(25,0)$ \\
$\begin{array}{l}\text { Utilização das tecnologias de informação e comunicação (à distância) no } \\
\text { ensino e na troca de informações entre profissionais }\end{array}$ & $6(16,6)$ \\
$\begin{array}{l}\text { Desenvolvimento de tecnologias para apoio ao enfrentamento da CovID-19 } \\
\text { Ações de capacitações aos estudantes e residentes para o enfrentamento } \\
\text { da COVID-19 }\end{array}$ & $5(13,8)$ \\
\hline
\end{tabular}

\section{Discussão}

Os resultados encontrados evidenciam a heterogeneidade da distribuição dos hospitais universitários, leitos gerais e leitos de UTI no Brasil. Diante da pandemia da COVID-19, essa situação é preocupante porque pode comprometer o atendimento, principalmente, a pacientes com sintomas mais graves. O cenário é pior para leitos de UTI e no Norte e Nordeste do país. Além disso, a presença de vazios assistenciais pode levar o sistema ao colapso, mesmo com taxas menos elevadas de infecção. ${ }^{(12)}$

A identificação das ações desenvolvidas demonstra a proatividade e rápida resposta dos HUFs no enfrentamento da pandemia de COVID-19. Embora as açóes desenvolvidas tenham sido classificadas nas dimensões Assistência, Gestão, Extensão e Ensino e Pesquisa, percebe-se uma complementa- ridade entre elas em prol da qualidade do cuidado em saúde, segurança do paciente e da equipe, bem como, o compromisso com a produçáo do conhecimento científico e tecnológico.

O novo coronavírus tem um comportamento ainda incompreendido pela comunidade acadêmica e científica, principalmente quanto às formas de transmissão. Nesse sentido, os dados encontrados em um estudo realizado em dois hospitais em Wuhan, China, alertaram sobre o risco potencial da transmissão pelo ar, visto que, foram encontradas partículas do vírus suspensas no ar em regióes adjacentes aos hospitais. ${ }^{(13)}$ A circulação de pessoas nessas regióes pode acarretar risco duplo de contaminação, uma pelo contato com pessoas potencialmente infectadas e a outra, pela exposição direta ao ar possivelmente contaminado. Assim sendo, destacaram-se as medidas adotadas pelos HUFs em relação à redução da circulação de pessoas no ambiente hospitalar. Açôes similares também foram desenvolvidas em hospitais chineses durante a pandemia. ${ }^{(14)}$

Aspecto inquietante de uma pandemia é o crescente fluxo de pacientes com consequente sobrecarrega dos sistemas de saúde, o que ocasiona uma escassez grave de recursos humanos e materiais nessas unidades. Tendo em vista as demandas assistenciais, tais recursos são tão importantes que não se deve poupar esforços para assegurar um quantitativo adequado desses elementos no cenário adverso de pandemia. Para isso, reorientar os moldes assistencialistas nas instituiçóes hospitalares requer maior rigidez no atendimento aos pacientes, organização das demandas primárias e suporte às demais necessidades decorrentes dos pontos de inflexão que porventura despontem na rotina diária. ${ }^{(15)}$

No âmbito da gestão, destacaram-se a contratação emergencial de novos profissionais e capacitação das equipes de saúde. A busca de novos profissionais é uma necessidade decorrente do aumento da demanda de pacientes por atendimento, principalmente para dar conta do pico da pandemia. Como foi observado na Itália e Espanha, os hospitais precisaram aumentar o seu quadro de profissionais e a sua capacidade de atendimento para evitar o colapso hospitalar diante do contexto pandêmico de infecções por Sars-CoV-2.(16) 
Como a COVID-19 era até então uma doença desconhecida, a realização de capacitações foi necessária para os profissionais de saúde que tem ou poderão ter contato com pessoas suspeitas e/ou infectadas com o novo coronavírus. Assim, fazem-se necessários treinamentos tanto sobre o manejo clínico da doença quanto para que permitam a efetivação prática da técnica adequada de paramentação e desparamentação, em prol da diminuição de risco de contaminaçáo e erros técnicos. ${ }^{(17)}$

Outra ação que merece destaque foi a adoção de medidas de suporte emocional aos profissionais, disponibilizando atendimento psicológico para os trabalhadores, com terapias complementares e atividades de relaxamento. Essa atenção especial à saúde mental dos profissionais é imprescindível em um momento de crescente carga de trabalho e tratamento de uma doença nova. Diante disso, os gestores e as lideranças institucionais devem incentivar os membros da equipe a pedir ajuda quando precisarem e envidar esforços no desenvolvimento de estratégias para ouvir e apoiar os profissionais diante momento desafiador. ${ }^{(7,18)}$

Além de açóes tangíveis para atender as suas preocupações, os profissionais de saúde desejam uma liderança visível durante esse período turbulento. Líderes, como administradores hospitalares, gerentes de enfermagem e chefes de divisão devem desenvolver maneiras inovadoras de estarem presentes e conectarem-se com suas equipes, dadas as restriçóes do distanciamento social. É essencial que os líderes entendam as fontes de preocupação dos profissionais de saúde e busquem abordagens que atenuem as preocupaçóes na medida do possível. ${ }^{(18)}$ Por isso, o desenvolvimento de campanhas nos HUFs para agradecimento aos profissionais da saúde, a elaboração de vídeos e mensagens de incentivo e apoio são importantes para motivar os profissionais nesse período táo delicado. Portanto, expressóes de gratidão pelo comprometimento dos profissionais de saúde e sua disposição de colocar-se em perigo para pacientes e colegas podem fazer a diferença neste momento desafiador. As lideranças da organização não precisam nem devem terceirizar a gratidão inteiramente ao público. ${ }^{(18,19)}$

Em relação às ações de extensão, destacou-se o trabalho dos HUFs na produção de materiais infor- mativos visando à prevenção da COVID-19, principalmente de forma on-line. Estas informaçôes podem contribuir com a saúde mental da população, visto que, estudos apontam que a enorme escala do avanço da epidemia está causando um grande impacto na saúde mental coletiva, podendo gerar e agravar sintomas de ansiedade, estresse, depressão, insônia, raiva e medo, de forma profunda e durável. Dessa forma, açóes educativas on-line podem representar um meio de interação e fonte de informação, contribuindo para o gerenciamento emocional. ${ }^{(19)}$

Outro destaque entre as ações de extensão foi a realização de campanhas para a arrecadação de insumos e doaçóes de Equipamentos de Proteção Individual (EPIs). Pode-se atribuir tal fato como resultado da escassez de insumos a nível mundial, visto que houve um aumento abrupto da procura de EPIs para prevenção da disseminação do vírus, não apenas por parte das instituiçôes de saúde, mas também da população em geral, ocasionando a falta para o uso dos profissionais da saúde. ${ }^{(20)}$

Quanto ao ensino e pesquisa, em consonância com as medidas gerais visando à redução de circulação de pessoas, ocorreu a suspensão de atividades relacionadas ao ensino e à pesquisa de graduação e pós-graduação. Porém, os hospitais universitários tradicionalmente caracterizam-se por estar na vanguarda na realizaçáo de pesquisas e no desenvolvimento de tecnologias em saúde. Desse modo, mesmo no início do período pandêmico, os achados deste estudo já indicam o comprometimento dos HUFs com a produção de novos conhecimentos sobre COVID-19.

As pesquisas que já vem sendo desenvolvidas sobre o novo coronavírus em outros países têm sido fundamentais para a melhoria da resposta à epidemia. ${ }^{(21)}$ No entanto, há uma necessidade contínua de maior investimento em investigaçóes científicas tanto para a produção de evidências para a prática clínica, quanto para o entendimento dos efeitos da COVID-19 na saúde mental da população e, principalmente, de grupos vulneráveis, como os profissionais da saúde. Para isso, pontua-se a importância da integração entre disciplinas numa perspectiva de colaboração internacional de produção de conhecimento e novas tecnologias. ${ }^{(22)}$ 
Como limitaçóes, é necessário registrar que o estudo foi realizado no início da pandemia de COVID-19 no Brasil e com base exclusivamente nas informações divulgadas nos sites dos hospitais universitários. Além disso, o levantamento foi restrito a HUFs, que não representam a maioria das instituiçôes hospitalares do País.

Apesar dessas limitaçóes, este estudo fornece um panorama das açôes desenvolvidas pelos HUFs no enfrentamento no início da pandemia de COVID-19 no Brasil. Também confere visibilidade ao protagonismo dessas instituiçôes, que por meio dos seus gestores estão desenvolvendo estratégias para o provimento das condiçóes e dos recursos necessários para o cumprimento da sua missão assistencial no contexto do Sistema Único de Saúde. Como sugestão para futuros estudos, pontua-se a importância da avaliação da repercussão das ações identificadas neste estudo no contexto organizacional, no ambiente de trabalho dos profissionais e na capacidade de atendimento dos hospitais universitários frente à demanda do sistema de saúde.

\section{Conclusão}

Os HUFs estão reorganizando o atendimento e buscando meios para prover as condiçôes necessárias para o enfrentamento da pandemia de COVID-19, a partir de açóes de assistência, gestão, extensão, ensino e pesquisa. $\mathrm{Na}$ assistência, destacou-se suspensão e/ou adiamento de consultas e atendimentos ambulatoriais, restrição e/ou suspensão de visitas e suspensão e/ou adiamento de cirurgias eletivas. Na gestão, as principais estratégias foram capacitação de profissionais para o manejo da COVID-19, contratação emergencial de profissionais da saúde e oferta de atendimento psicológico para os trabalhadores. $\mathrm{Na}$ extensão, a principal frente de atuação foi a elaboração de materiais educativos sobre medidas de prevenção. No campo do ensino e da pesquisa, houve suspensão de atividades relacionadas à graduação e pós-graduação, porém tiveram início novos projetos de pesquisa relacionados à COVID-19.

\section{Agradecimentos}

Ao Conselho Nacional de Desenvolvimento Científico e Tecnológico $(\mathrm{CNPq})$, processo $\mathrm{n}^{\mathrm{o}}$ 402392/2020-5 e à Fundação de Amparo à Pesquisa e Inovação do Estado de Santa Catarina (Fapesc), protocolo $\mathrm{n}^{\mathrm{o}}$ : PEC2020111000005. O presente trabalho contou com apoio da Coordenação de Aperfeiçoamento de Pessoal de Nível Superior Brasil (CAPES) - Código de Financiamento 001.

\section{Colaborações}

Santos JLG, Lanzoni GMM, Costa MFBNA, Debetio JO, Sousa LP, Santos LS, Marcelino TB, Mello ALSF contribuíram com a concepção do projeto, análise e interpretação dos dados, redação do artigo, revisão crítica relevante do conteúdo intelectual e aprovação final da versão a ser publicada.

\section{Referências}

1. World Health Organization (WHO). Coronavirus disease 2019 (COVID-19) - Situation Report 56. Geneva: WHO; 2020 [cited 2020 May 29]. Available from: https://www.who.int/docs/default-source/ coronaviruse/situation-reports/20200311-sitrep-51-covid-19.pdf

2. Chen Y, Liu Q, Guo D. Emerging coronaviruses: genome structure, replication, and pathogenesis. J Med Virol. 2020 Apr;92(4):418-23.

3. Medeiros EA. Health professionals fight against COVID-19. Acta Paul Enferm. 2020;33: e-EDT20200003.

4. Rodriguez-Morales AJ, Gallego V, Escalera-Antezana JP, Méndez CA, Zambrano LI, Franco-Paredes C, et al. COVID-19 in Latin America: the implications of the first confirmed case in Brazil. Travel Med Infect Dis. 2020;35:101613.

5. Oliveira AC, Lucas TC, Iquiapaza RA. What has the covid-19 pandemic taught us about adopting preventive measures? Texto Contexto Enferm. 2020;29:e20200106.

6. Wang H, Wang S, Yu K. COVID-19 infection epidemic: the medical management strategies in Heilongjiang Province, China. Crit Care. $2020 \mathrm{Mar} ; 24(1): 107$

7. Stoller JK. Reflections on leadership in the time of COVID-19. BMJ Leader Stoller JK. BMJ Leader. 2020;4(2):77-9.

8. Empresa Brasileira de Serviços Hospitalares (EBSERH). Sobre os Hospitais Universitários Federais [Internet]. Brasília (DF): EBSERH; 2020. [citado 2020 Jun 8]. Disponível em:https://www.gov.br/ebserh/ pt-br/acesso-a-informacao/hospitais-universitarios-federais/sobreos-hospitais-universitarios-federais

9. Medeiros EA. Desafios para o enfrentamento da pandemia covid-19 em hospitais universitários. Rev Paul Pediatr. 2020;38:e2020086. 
10. Di Gennaro F, Pizzol D, Marotta C,Antunes M, Racalbuto V, Veronese N, et al. Coronavirus diseases (COVID-19) current status and future perspectives: a narrative review. Int J Environ Res Public Health. 2020 ;17(8):2690.

11. Shen Y, Cui Y, Li N, Tian C, Chen M, Zhang YW, et al. Emergency responses to Covid-19 outbreak: experiences and lessons from a general hospital in Nanjing, China. Cardiovasc Intervent Radiol. 2020;43(6):810-9.

12. Noronha KV, Guedes GR, Turra CM, Andrade MV, Botega L, Nogueira D, et al. Pandemia por COVID-19 no Brasil: análise da demanda e da oferta de leitos hospitalares e equipamentos de ventilação assistida segundo diferentes cenários. Cad Saude Publica. 2020;36(6):e00115320.

13. Liu Y, Ning Z, Chen Y, Guo M, Liu Y, Gali NK, et al. Aerodynamic analysis of SARS-CoV-2 in two Wuhan hospitals. Nature. 2020;582(7813):557-60.

14. Jin X, Lian JS, Hu JH, Gao J, Zheng L, Zhang YM, et al. Epidemiological, clinical and virological characteristics of 74 cases of coronavirusinfected disease 2019 (COVID-19) with gastrointestinal symptoms. Gut. 2020;69(6):1002-9.

15. Chen T, Wang Y, Hua L. "Pairing assistance": the effective way to solve the breakdown of health services system caused by COVID-19 pandemic. Int J Equity Health. 2020;19(1):68-72.

16. Ceylan Z. Estimation of COVID-19 prevalence in Italy, Spain, and France. Sci Total Environ. 2020;729:0048-9697.
17. Oliveira HC, Souza LC, Leite TC, Campos JF. Personal Protective Equipment in the coronavirus pandemic: training with Rapid Cycle Deliberate Practice. Rev Bras Enferm. 2020 ;73(73 Suppl 2):e20200303.

18. Shanafelt T, Ripp J, Trockel M. Understanding and Addressing Sources of Anxiety Among Health Care Professionals During the COVID-19 Pandemic. JAMA. 2020;323(21):2133.

19. Turabian JL. Implications on mental health by the coronavirus disease 2019 (COVID-19) pandemic: The role of general practitioner. Arch Psychiatr Ment Health. 2020; 4:35041.

20. Camargo MC, Lima AA, Bastos BP, Santos DL, Mota SM, Silva RB, et al. Eficácia da máscara facial (TNT) na população para prevenção de infecções por coronavírus: revisão sistemática. Ciênc Saúde Coletiva [Internet]. 2020 [citado 2020 Jun 8]. Disponível em: http://www. cienciaesaudecoletiva.com.br/artigos/eficacia-da-mascara-facial-tntna-populacao-para-prevencao-de-infeccoes-por-coronavirus-revisaosistematica/ $17578 ?$ id $=17578$

21. Lipsitch M, Swerdlow DL, Finelli L. Defining the Epidemiology of Covid-19 - Studies Needed. N Engl J Med. 2020;382(13):1194-6.

22. Holmes EA, O'Connor RC, Perry VH, Tracey I, Wessely S, Arseneault L, et al. Multidisciplinary research priorities for the COVID-19 pandemic: a call for action for mental health science. Lancet Psychiatry. 2020;7(6):547-60. 